\title{
EL YACIMIENTO DE CABO BUSTO (VALDÉS, ASTURIAS). UNA SECUENCIA DEL PLEISTOCENO MEDIO EN EL NORTE PENINSULAR
}

\author{
THE CABO BUSTO SITE (VALDÉS, ASTURIAS). A MIDDLE PLEISTOCENE \\ SEQUENCE IN THE NORTH OF THE IBERIAN PENINSULA
}

por

JOSÉ ADOLFO RODRÍGUEZ ASENSIO*

\begin{abstract}
"Les chiffres obtenus sont immenses, comparés à ceux de l'Histoire et si nous essayons de suivre dans ce lointain passé, les pas de l'humanité naissante, nous n'avons plus pour nous renseigner que le témoignage des pierres taillés."
\end{abstract}

H. Breuil et G. Zbyszewski (1945): Contribution á l'étude des industries paléolithiques du Portugal et de leurs rapports avec la géologie du Quaternaire. Vol. II. Pág. 590.

RESUMEN El yacimiento de cabo Busto en el concejo asturiano de Valdés muestra, tras las excavaciones llevadas a cabo desde 1992, una estratigrafía que va desde el Pleistoceno medio hasta los albores de la glaciación würmiense y que desde el punto de vista cultural aporta interesantes datos para la reconstrucción de los primeros habitantes de Asturias cuya llegada a estas tierras, tras estos estudios, debe ser situada en épocas mas antiguas de las hasta ahora reconocidas por la arqueología prehistórica. Paleoculturas del Pleistoceno medio y Achelense superior son los dos momentos culturales que están representados en este yacimiento.

\begin{abstract}
The Cape Busto site in the municipality of Valdés in Asturias, under excavation since 1992, shows a stratigraph which goes from the middle Pleistocene Period to the dawning of the Würmien Glaciation. From the cultural perspective it contributes interesting data for the reconstruction of the first inhabitants of Asturias whose arrival to these lands, after these investigations, must now be placed in temps most ancients than up till now for prehistoric archaeological. The two cultural moments represented in this deposit are the middle Pleistocene Period and the upper Acheulean.
\end{abstract}

* Área de Prehistoria. Facultad de Geografía e Historia. Universidad de Oviedo. Tnte. Alfonso Martínez s/n. 33011 Oviedo. Adolfo@sci.cpd.uniovi.es 


\section{INTRODUCCIÓN}

El yacimiento de cabo Busto fue descubierto por el Dr. José Manuel González y Fernández Valles, dado a conocer en 1968 y valorado inicialmente por nosotros (Rodríguez Asensio 1976 y 1983) tras un análisis de los materiales líticos recogidos en superficie en diferentes ocasiones desde su descubrimiento, por lo que dicho yacimiento pasó a la literatura de los estudios prehistóricos en Asturias, en la década de los 80, como uno de los puntos con interés en excavaciones futuras. Ya apuntábamos en 1983 que posiblemente pudiera encontrarse en el cabo Busto una estratigrafía que nos permitiese fortalecer algunas de las hipótesis expuestas en aquella época.

Se sitúa el sitio de Busto en la plataforma del mismo nombre, que en esta zona del concejo de Valdés se encuentra a una altitud de $63 \mathrm{~m}$ sobre el nivel actual del mar. Se localiza entre los $43^{\circ} 34^{\prime} 10^{\prime \prime}$ latitud N. y $6^{\circ} 29^{\prime} 50^{\prime \prime}$ longitud W., y es uno de los salientes mas septentrionales, junto con los cabos Vidío y Peñas, de Asturias. La plataforma del cabo Busto está formada por un aplanamiento liso y ancho, como es la Rasa litoral cantábrica, con una anchura en esta zona que oscila entre $750 \mathrm{~m}$. y $3 \mathrm{kms}$, y limita en el interior con los relieves más pronunciados, a los que se adosa, que se interpretan como acantilados de la antigua línea de costa.

Ya en nuestro trabajo de síntesis del paleolítico inferior (Rodríguez Asensio 1983) definíamos la Rasa litoral cantábrica como una de las zonas de habitabilidad mas importante del hombre prehistórico en estos primeros momentos de ocupación de las tierras astures. En ella se encuentran importantes conjuntos líticos, algunos de los cuales nos han aportado las únicas estratigrafías con las que se cuenta en estos momentos para intentar la comprensión global del paleolítico antiguo en esta zona.

En este sentido, podemos recordar algunos de los lugares con materiales líticos en la rasa litoral cantábrica. Desde la orilla del Eo hasta la región de Peñas, se encuentran varios yacimiento y asentamientos además de Busto, como Arnao, Villadún, Cornayo, Salave, Caroyas, Salamir (Rodríguez Asensio 1983), Folgueiron, Cotarelo y los abrigos de Peña Caldeira (Maradona y Martínez Faedo 1995), Ribón (Noval Fonseca 1996), L'Atalaya (Díaz Nosty y Sierra Piedra 1995), Santa María del Mar (Pérez Pérez y González Menéndez 1990), Pinos Altos y Las Arribas en San Martín de Laspra (Pérez Pérez y González Menéndez 1991), Arroyo de Caseras, que anteriormente se había dado a conocer como Foncubierta (González 1968 y Rodríguez Asensio 1983). Ya en la región del cabo Peñas que apuntamos como un "territorio de caza", se encuentran sitios con materiales líticos o yacimientos como Verdicio, El Otero, plataforma de Peñas, Bañugues, Punta la Vaca, Moniello, Aramar, Antromero, L'Atalaya (Rodríguez Asensio 1978, 1980, 1983; Rodríguez Asensio y Flor Rodríguez 1980, 1983; Pérez Pérez, 1975. Pérez Pérez y González Menéndez 1996), por citar únicamente los mas representativos.

A la margen oriental de la región de Peñas, en el concejo de Gijón, se citan desde antiguo (Gómez de Llarena 1933) materiales de tipología de paleolítico inferior, y recientemente nosotros mismos hemos hecho una síntesis en la que se citan todos los materiales encuadrables en esta clasificación prehistórica (Rodríguez Asensio 1995). Zonas del concejo de Gijón como Campa Torres, Tremañes, Roces y Viesques, recientemente las hemos considerado como aglutinantes de varios lugares en los que han aparecido materiales líticos de tipologías inferopaleolíticas en un intento de comprensión global del poblamiento más antiguo conocido en esta zona de la costa central asturiana (Rodríguez Asensio y Noval Fonseca 1997 e. p.). Por último, citaremos un bifaz aislado encontrado en Cancienes, concejo de Corvera (Montes Barquín y Fernández Ramos 1997 e. p.) (fig. 1).

En el caso de cabo Busto hemos tenido la suerte de poder determinar y definir una estratigrafía que, aunque presenta no pocos interrogantes desde el punto de vista sedimentológico y arqueológico, creemos puede ser válida para el Pleistoceno medio y superior en el norte peninsular. La existencia de niveles fértiles arqueológicos y sus interesantes conclusiones se suman a la no menos interesante cuestión del origen y 
formación de la Rasa litoral que desde su definición mantiene estos interrogantes sin una respuesta clara, con opiniones contrapuestas.

\section{ESTRATIGRAFÍA}

En las excavaciones realizadas desde 1992 hasta 1997 logramos definir una estratigrafía completa, que sedimentológicamente abarca desde la capa húmica del suelo actual hasta las cuarcitas de la propia rasa litoral, con niveles arqueológicamente fértiles. La secuencia estratigráfica que damos como definitiva ha sido expuesta por nosotros (Rodríguez Asensio 1996) en una aproximación de estudio.

La zona de cabo Busto se incluye en el Mapa de suelos de Asturias (Guitian et al. 1985) como "tierra parda podsolizada", dentro de los suelos bien drenados, y como "podsol húmico" (Álvarez et al. 1995), que se asientan sobre las cuarcitas de la "serie de los Cabos", que son el principal material sobre el que se ha formado la Rasa litoral en esta zona. También se indican zonas de "turbera" en la plataforma de Busto.

De techo a muro la estratigrafía definida en las excavaciones es la siguiente (fig. 2):

\section{Nivel I:}

Suelo vegetal de color negro intenso muy enriquecido con materia orgánica. Internamente, según la intensidad de la coloración, se puede dividir en dos subniveles, $a$ y $b$, siendo el $a$ el superior, de un tono menos intenso que el $b$ o inferior. Se trata de un suelo húmico que se ha enriquecido con las plantaciones de pinares que en esta zona se realizaron hasta hace no muchos años y de las cuales aun encontramos restos en las excavaciones arqueológicas. Es un suelo estéril arqueológicamente. Tiene $45 \mathrm{~cm}$. de espesor.

\section{Nivel II:}

Suelo fértil arqueológico. Dentro de una matriz limosa se encuentra gran acumulación de cantos, cantos rotos, fragmentos de rocas y materiales prehistóricos que dan un aspecto de "pedrero" una vez limpiada y retirada la matriz. Muestra un grosor de $22 \mathrm{cms}$. aunque su interpretación sedimentológica apunta a que este nivel habría sido más grueso, con diferencias interiores y posiblemente con momentos distintos de ocupación prehistórica. Posteriormente, este nivel sufrió un fuerte lavado y el material fino fue decantándose, de tal manera que se fue adelgazando hasta llegar al grosor actual, que seguramente es bastante menor del que tenía en el momento de formación. Esto nos hace pensar que lo que aparentemente hoy es un suelo de ocupación único puede haber sido un nivel con una ocupación más larga y duradera.

La coloración de este nivel es algo negruzca, lo que seguramente se debe al lavado del nivel I, de gran aporte orgánico, como se ha indicado mas arriba.

La presencia de abundantes cantos y fragmentos de cantos, tabletas etc. junto a los cuales y entre ellos se encuentran los materiales prehistóricos, es uno de los interrogantes planteados, que esperamos poder resolver en las próximas campañas. Todos estos materiales líticos, prehistóricos o no, tallados o no, se encuentran "in situ"?, ¿han sido llevados hasta este lugar desde otras zonas cercanas, posiblemente desde alguna de las playas colindantes y se muestran como auténtica "cantera" de materia prima para la confección de los instrumentos prehistóricos? Estas preguntas y otras en esta misma línea se nos presentan ante el nivel II y su valoración. 
Las piezas líticas prehistóricas muestran diferentes pátinas, aunque siempre dentro de valores de intensidad mediana y no alteradas por posibles rodamientos, lo cual nos hace pensar que se trata de restos dejados en el lugar. El estudio de estos materiales prehistóricos no se realizará en su totalidad y en profundidad hasta que las excavaciones lleguen a su final. No obstante, las primeras valoraciones (Rodríguez Asensio 1995) sitúan estos restos dentro de los niveles culturales de Achelense superior y apuntan a que posiblemente se trate de un asentamiento, como ya hemos dicho, duradero.

¿Existe la posibilidad de encontrar alguna estructura de este asentamiento o algún resto, además de los útiles prehistóricos, como "manuports" que nos lleven más certeramente por el camino de la interpretación de un asentamiento?

\section{Nivel III:}

Suelo de $41 \mathrm{cms}$ de grosor que además de sus características propias, como son, fundamentalmente, la de estar integrado y compuesto de gravillas y cantos pequeños, se define nítidamente por encontrarse entre el nivel II, fértil arqueológicamente como se ha descrito anteriormente, y el nivel IV, que juntamente con el nivel $\mathrm{V}$, como se analiza más adelante, forman un conjunto geológico característico.

En este nivel se pueden ver claramente "bolsadas" en forma de cuña, intercaladas de techo a muro, que pueden ser interpretadas como acuñaciones producidas por el hielo. Están formadas por cantos de tamaño pequeño y gravillas y entre ellas hemos encontrado alguna pieza lítica tallada (útiles en lasca y lascas).

No obstante, aunque este nivel no ha sido excavado en extensión y por tanto las conclusiones a las que se pueden llegar en estos momentos han de sujetarse a las lógicas revisiones y análisis críticos que deberán realizarse tras la excavación del mismo, hemos planteado como interrogante prioritario durante estos trabajos el poder definir si los materiales antrópicos prehistóricos que se encuentran en el mismo, forman parte de las mencionadas "cuñas de hielo" y por tanto serían del nivel II, o por el contrario estamos ante la posibilidad de ver este nivel III como un suelo diferente en el que se encuentran restos de ocupación humana atestiguados por los restos líticos citados.

Es prematuro hablar de diferencias tecnológicas y tipológicas o de pátina o de cualquier otra variable en los materiales líticos de ambos niveles, entre otras cuestiones porque los aparecidos en el nivel III son muy poco numerosos.

\section{Nivel IV:}

Este nivel de arenas, juntamente con el nivel $\mathrm{V}$ que se describe posteriormente, lo entendemos como una unidad sedimentológica, en el sentido de considerar ambos como restos de aporte fluvial. Muestra un grosor de $84 \mathrm{cms}$.

A la espera de los correspondientes análisis sedimentológicos, precisos y detallados, diremos que en este nivel de arenas se diferencian varios momentos de sedimentación que han dejado diversas tonalidades dentro de una gama de grises, más o menos claras. En varios casos se aprecia algún encostramiento intercalado.

\section{Nivel V:}

Entre el nivel IV y las cuarcitas de la Rasa litoral que aparecen a $59 \mathrm{cms}$ por debajo de éste, se encuentra un nivel de cantos rodados muy compactados y cementados entre sí, con encostramientos intercalados similares 
a los existentes en el nivel IV. Los cantos, de tamaño medio y pequeño son de cuarcita de grano fino de color grisáceo. Material éste en el que se han tallado muy pocos instrumentos líticos en los momentos posteriores y' representados, como se ha dicho, en los niveles II y III. Los niveles IV y V, que con anterioridad habíamos subdividido sedimentológicamente pueden diferenciarse con mayor precisión. No obstante, desde el punto de vista arqueológico consideramos válida la división en dos niveles, que diferenciamos por los materiales que los componen: arenas el IV y cantos el V.

Este nivel V se distingue fácilmente con el IV en el acantilado, a pocos metros de distancia del lugar de la excavación. De él se ha extraído un canto trabajado de cuarcita (Rodríguez Asensio 1995) que evidencia, sin que por el momento parezca que puedan existir otras explicaciones razonables, la existencia de restos industriales en momentos más antiguos que, hoy por hoy, se nos muestran difíciles de encuadrar cronológicamente, pero que a modo de mera hipótesis de trabajo podemos intentar, y así lo hemos hecho, aventurando una aproximación cronológica cultural.

\section{Nivel VI:}

Cuarcitas de la "Serie de los Cabos" (Marcos 1976)

\section{INTERPRETACIÓN}

Aunque no se cuenta con todos los datos necesarios para poder hacer una interpretación completa del yacimiento de Busto, ya que están en estudio los materiales prehistóricos, los sedimentológicos y aún restan por realizar los correspondientes a la luminiscencia, gracias a dos sensores que se encuentran enterrados en los niveles fértiles, creemos que se pueden adelantar determinadas conclusiones que, siempre sujetas a posteriores revisiones o matizaciones, son las que a la vista de los resultados existentes hasta el momento presente nos permiten intentar la reconstrucción, aunque sea mínima, del más antiguo poblamiento humano que se conoce en el norte de la península ibérica.

A la vista de la estratigrafía propuesta en las páginas precedentes elevamos a definitivas, las siguientes conclusiones:

1.- Existencia de un asentamiento humano cuyas huellas se encuentran en el nivel II y que hemos interpretado (Rodríguez Asensio 1996) como Achelense superior, aunque apuntamos también la posibilidad de que este nivel sea el resultante de uno más grueso conseguido por el aporte de materiales de épocas más amplias y que ahora después del intenso lavado de material fino y de su matriz, se nos muestra más fino y con una gran concentración de material grueso. Si esta interpretación es correcta estaríamos ante la posibilidad de que no todos los útiles prehistóricos del mismo perteneciesen al mismo momento, sino que hayan existido varios y diferentes momentos de ocupación. No obstante, el análisis interno del nivel II no nos ha permitido diferenciar estos posibles momentos sucesivos de ocupación humana.

Esta posibilidad no se puede, hoy por hoy, considerar como cerrada si se tiene en cuenta el análisis de los materiales líticos, pues por tipología y tecnología no parecen existir demasiadas diferencias internas que nos indiquen distintos momentos culturales.

Sí se puede apreciar en la pátina una diferencia en intensidad en algunas piezas prehistóricas. Somos conscientes, sin embargo, de que el análisis de las pátinas ha de ser tenido en cuenta de la manera más cauta posible, pues ésta se va formando con diversos grados de intensidad no solo por cuestiones de cronología sino porque también en ella influyen, por ejemplo, el tipo de materia en el que está realizado el útil, la 
exposición a los elementos atmosféricos o la simple composición química de la matriz del suelo en el que está incluida y que en ocasiones puede variar mucho en espacios cortos.

Este asentamiento humano del achelense superior, cronológicamente lo hemos incluido en el interglaciar Riss-Wurm y que continúa en el Wurm I, correspondiente al isótopo 5 (128.000 - 71.000), con una fecha absoluta que ciframos en torno a 100.000 años, si tenemos en cuenta el yacimiento de la cueva del Castillo en Cantabria (Cabrera 1984) y nuestros propios resultados y propuestas para el paleolítico inferior en Asturias con lugares de referencia como Bañugues (Rodríguez Asensio 1978, 1980; Rodríguez Asensio \& Noval 1997), Louselas (Rodríguez Asensio 1997 e.p.) en la costa o Paredes (Rodríguez Asensio 1983; Noval 1995) y Llagú (Rodríguez Asensio 1983) en las tierras interiores asturianas (fig.3).

Representativo resulta el grupo de los bifaces (amigdaloides, protolimandes, triangulares, subtriangulares, subcordiformes...), muchos de ellos en grandes lascas y de los hendidores (tipos $\mathrm{O}, 1$, 2, 2-3) que junto a los escasos picos triedros forman la colección de útiles masivos. La escasez de "cantos trabajados" $(1 \%)$ en cualquiera de sus variantes nos indica que nos encontramos ante una cadena operativa bifacial y ante una cadena operativa de producción de soportes primarios, y no de producción de cantos y además, consideramos este hecho muy importante a la hora de las interpretaciones generales del paleolítico antiguo en el norte peninsular. Hasta ahora se había concedido demasiada importancia, desde nuestro opinión, a la presencia de "cantos trabajados", sobre todo en las "recogidas en superficie". Mantenemos serias dudas sobre la talla y sobre la cronología. Muchas de las colecciones de "cantos" se nutren de materiales no prehistóricos, tallados en momentos históricos, incluso actuales, como se ha puesto de manifiesto recientemente, con una claridad meridiana (Cano Pan y Vázquez Varela 1996) y, además, muchos de los "cantos" no están tallados, sino que presentan seudolevantamientos producidos por fenómenos de rodamiento y golpeo entre sí.

Entre los útiles en lasca, ya destacamos el grupo de las raederas y las muescas, con valores porcentuales muy distantes de otros útiles que aunque se encuentran representados no lo son significativamente. Los núcleo pequeños, algunos de técnicas de preparación, los restos de talla y las lascas abundantes nos sugieren las dos cadenas operativas apuntadas, la bifacial y la de producción de soportes primarios. Las zonas de cantera, que serían las cercanas playas de la zona, aportarían los cantos y bloques y en ellas se realizaría el trabajo elemental de la extracción de las grandes lascas en las que posteriormente se tallarán los útiles masivos (bifaces, hendidores, picos triedros). Estas grandes lascas y cantos mas pequeños serán trasladados al lugar del yacimiento, posiblemente asentamiento, y allí se procederá a la realización de los siguientes pasos de las cadenaś operativas apuntadas. La existencia de restos de talla, núcleos y lascas, lo atestigua.

La primera valoración tecnológica y tipológica de los materiales de este nivel achelense (Rodríguez Asensio 1995) nos ha permitido definir una cadena lítica que se nos muestra con una cierta simplicidad en cuanto a los procesos técnicos de producción, una alta uniformidad con escasa variabilidad técnica y morfológica y baja especialización funcional. La inmediatez de las estrategias en la transformación y uso de los elementos líticos y el localismo en la captación y distribución de materias primas son las características fundamentales del conjunto lítico achelense de Busto (fig.4).

2.- Los restos industriales en el nivel III que apuntamos como posibles muestras de algún momento cronológico y cultural intermedio entre el nivel achelense (II) y el nivel de paleoculturas del nivel V, hoy los interpretamos como pertenecientes al mismo nivel achelense superior que han sido absorbidos por "cuñas de hielo" hacia abajo, de tal manera que esta sería la explicación de la existencia de materiales líticos prehistóricos en el nivel III. No descartamos sin embargo ocupaciones humanas en momentos anteriores al achelense superior, y en esta línea parece deban interpretarse algunos restos líticos encontrados en otros sitios del solar astur, pero en Busto pensamos que esta interpretación que ya apuntábamos como posible (Rodríguez Asensio 1996) de la absorción de determinados materiales por las cuñas de hielo, es la más 
plausible. Esta evidencia de fenómenos producidos por el hielo-deshielo (crioturbación) se encuentra muy bien representada en el nivel II, sobre todo en la base, y así en las excavaciones de la campaña de 1997 hemos rescatado de este nivel un bifaz tallado, posteriormente crioturbado y sus tres fragmentos desplazados varios centímetros entre sí.

3.- Restos de una industria anterior que estratigráficamente se puede asignar a momentos más antiguos y que hemos propuesto pertenecientes al Pleistoceno medio en general, aunque serán necesarios más datos y análisis para poder aquilatar con precisión esta primera aproximación.

Se trata del canto tallado aparecido y extraído del interior del nivel V. Es un canto de cuarcita de grano fino, aplanado, de $5 \mathrm{kgs}$. de peso y $230 \times 230 \times 100 \mathrm{mms}$. de dimensiones máximas. Muestra varios levantamientos en uno de sus bordes bifacialmente, distribuyéndose cuatro en una cara y tres en la opuesta, lo que da una zona de borde cortante. Lo hemos interpretado como un núcleo de lascas a la vista de las dimensiones y del peso. Tampoco nos ofrece duda en cuanto a la talla se refiere, una lasca de decorticado primario con posteriores levantamientos para lograr una zona apuntada.

La asignación cronológica y cultural de estos útiles y del nivel en el que se encuentra no resulta fácil. No obstante, pensamos que sí se puede intentar una aproximación a ella, a través de la deducción de los datos aportados por el estudio de la zona donde se encuentran los depósitos en cuestión, es decir la Rasa litoral cantábrica.

Abundante es la bibliografía que plantea los aspectos cronológicos de la Rasa litoral que juntamente con el tema de su formación, son los dos problemas que más han dado que hablar y más opiniones encontradas han provocado, desde quienes apuestan por el origen marino de esta plataforma, puesto de manifiesto por primera vez por Barrois (1882), hasta quienes defienden el origen continental, expuesto por primera vez por E. Hernández Pacheco (1930 y ss.), sin olvidar a quienes intentan unir ambas teorías, como Guilcher (1974), quien afirma que las rasas no tienen un origen único.

F. Hernández Pacheco es el primero que habla de formación fluvial para los sedimentos encontrados sobre estas superficies y juntamente con Asensio Amor (1959-64) afirma que los sedimentos son de origen continental "eliminando en cuanto a su procedencia se refiere cualquier aportación que el mar pudiera haber realizado".

Nosotros mismos (Rodríguez Asensio 1983) hemos analizado la bibliografía más importante sobre este tema, con vistas a los posibles resultados e implicaciones que pudiera tener con las industrias encontradas en la misma, y los problemas que las rodean, desde la zona de Peñas hasta la costa lucense, en Galicia. G. Flor (1983) realiza un completo estudio sobre la correlación entre las diferentes superficies de arrasamiento marino del borde costero asturiano y divide estas plataformas por sectores geográficos en función de sus altitudes actuales que van desde los $264 \mathrm{~m}$. de la Rasa I hasta los 5-6 m. de las Rasa VII. Según esta clasificación, la rasa a la que nos estamos refiriendo y sobre la que se encuentran los depósitos por nosotros estudiados sería la IV, que según este autor (Flor 1983) llega a solaparse con la III en el occidente.

La plataforma de Busto actualmente se encuentra a $63 \mathrm{~m}$. sobre el nivel del mar y si aceptamos que los depósitos de cantos y arenas existentes en los niveles IV y $\mathrm{V}$ son fluviales, como parece indicar su interpretación, hemos de buscar unos momentos en los que el nivel del mar se encontrase a la altitud de la rasa litoral. Aunque quedan aspectos en este tema que están sujetos a discusión y no todos aceptan la teoría del origen marino de la citada rasa, nosotros pensamos que es válida esta interpretación a tenor de los depósitos existentes así como de las ocupaciones humanas de estos espacios.

La asignación cronológica ha sido uno de los aspectos más debatidos y, si analizamos la bibliografía más importante al respecto, encontramos que los autores que asignan una edad al aplanamiento que se encuentra en cabo Busto, están representados en un primer momento por Gómez de Llarena y Royo Gómez (1927) que sitúan en el Siciliense la altitud de 85 - 95 m (cabo Vidrias, cabo Peñas, Ribadesella....) y en el Milaciense 
la altitud de 50-60 m. (las más extendidas desde el occidente asturiano), retrasando al Plioceno el nivel de 120 - $130 \mathrm{~m}$., mientras que los bajos niveles que se encuentran a $10-20 \mathrm{~m}$. pertenecerían, según estos autores, al Monastiriense.

En 1957 con motivo del V Congreso Internacional de I.N.Q.U.A. Llopis y Jordá admiten como Birot y Solé (1954) edad tirreniense sin más precisiones para la rasa elevada, que sitúan a $120 \mathrm{~m}$. y tirreniense II sería la edad asignada a la rasa de $50-60 \mathrm{~m}$.

En 1963 Llopis explica el mismo esquema cronológico que en 1957, esta vez referido a la zona de Luarca, y asigna a la Rasa de 60-100 m. edad Tirreniense, a la representada en los niveles de 2 - $3 \mathrm{~m}$. Tirreniense III y la zona intermedia, de 15-20 m. al Tirreniense II. La regresión Riss sería la responsable de haber generado la rasa de 60-100 m., la Würm, la de 15-20 m., mientras que en el Tirreniense se produce una interrupción por la transgresión flandriense.

Nonn (1966) opina que la rasa occidental ya existía y era ocupada por el mar en el Tirreniense I. El nivel Tirreniense II está representado en la ribera de Ribadeo, siendo mas antiguo que el interglaciar Riss-Würm. También Mary (1971) se inclina por una edad Tirreniense para los niveles bajos entre 30 y $7 \mathrm{~m}$. La rasa de $100 \mathrm{~m}$. para este autor debió formarse a comienzos del Cuaternario y mas probablemente en el Plioceno.

Para Asensio Amor (1970) la rasa sería el resultado de una superficie o glacis de erosión formada al final del Plioceno. En el Pleistoceno antiguo sería remodelada por acciones torrenciales y marinas y posteriormente retocada por las nuevas transgresiones marinas; es decir, en tiempos del interglaciar MindelRiss, una nueva transgresión marina alcanza altitudes de 35-40 m. Posteriormente la retirada del mar en el periodo glaciar rissiense y en el interglaciar Riss-Würm tiene lugar una nueva transgresión y progresiva modificación de la rasa

En términos generales, e insistimos en que quedan muchos aspectos sin aclarar, se puede apuntar como resumen y a modo de conclusión no definitiva pero sí muy probable, que, una vez arrasada la plataforma en momentos pliocenos y tras las transgresiones habidas en el Pleistoceno inferior que podrían haber llegado a situar el mar a $100 \mathrm{~m}$., parece haberse dado una transgresión, posiblemente la última por encima de los niveles de cabo Busto, a principios del Pleistoceno medio entre 800.000 y 600.000 años, que se correspondería con los primeros momentos del Tirreniense o finales del Siciliense y, desde el punto de vista del esquema glaciar, con el interglaciar Mindel - Riss, en el primer caso, o Gunz - Mindel, en el supuesto de que sea aceptada la segunda hipótesis, y por tanto de mayor antigüedad.

Será en estos momentos cuando el aporte fluvial causante de los depósitos de cantos del nivel $V$ se encuentre a nivel del mar y antes, por tanto, de que se encaje la red fluvial existente en la zona. Este momento que parece, según opinión geológica, tal como hemos visto más arriba, perteneciente a los inicios de la transgresión tirreniense, del que desconocemos su duración en el tiempo, en alguno de sus momentos habrá arrastrado materiales líticos manufacturados por el hombre.

Este segundo nivel de ocupación humana estratigráficamente, aunque sería el primero cronológicamente, es muy difícil de situar. Si la interpretación fluvial de los sedimentos del nivel V es acertada y así lo creemos, esto nos llevaría a un momento de máxima extensión oceánica que haya permitido que estos grandes y potentes aportes fluviales se entregaran cómodamente al mar. Piénsese que la altitud de la Rasa en Busto es de 60 $\mathrm{m}$. y aunque pueden haber existido fenómenos epirogenéticos en la configuración actual, es indiscutible que la existencia de depósitos fluviales a $60 \mathrm{~m}$. de altitud nos indica, en primer lugar, un aumento oceánico importante, y en segundo, que la línea de costa deberá situarse varias centenas (varios kilómetros) de metros más hacia el interior del mar actual.

Según los estudios de la proporción de O18 existente en los sedimentos marinos el momento de máxima extensión oceánica y por tanto de más alto nivel marino, en los últimos 300.000 años se ha producido en el primer momento del interglaciar Riss-Würm, es decir en la fase isotópica 5e, cuya cronología absoluta se situaría en torno a 128.000 años. No obstante, descartamos esta posibilidad de interpretación cronológica 
para el nivel V de Busto al considerarla demasiado moderna para el aporte fluvial en esta zona, que se entiende anterior al encajamiento de la red. Pensar que ésta se haya encajado a lo largo de la última glaciación es excesivamente aventurado con los datos sedimentológicos actuales y, aunque la descartamos, hemos de seguir manteniendo los estudios y análisis interpretativos de esta plataforma cuyas respuestas a los interrogantes más importantes se siguen resistiendo.

Momentos de importante extensión oceánica se han dado en las fases isotópicas 7, de 245.000 a 186.000; 9, de 339.000 a $303.000 ; 11$, de 423.000 a $362.000,13$, de 524.000 a 478.000 , fundamentalmente, pues los máximos oceánicos conseguidos en momentos anteriores y coincidentes con las fases isotópicas 15,17 y 19 , hasta llegar al comienzo del pleistoceno medio, en el paso de la polaridad inversa Matuyama a la polaridad normal Brunhes, son menos intensas y por tanto con avances marinos de menor intensidad.

¿En qué momento, pues, de las fases 7, 9, 11 ó 13 se ha podido producir la sedimentación del nivel V que presenta industria lítica? Como hipótesis de trabajo hemos situado el límite más allá del cual no creemos posible esta sedimentación al comienzo de la fase 13 (Rodríguez Asensio 1996), que de manera más global y comprensible colocamos en el medio millón de años.

El encaje de la red fluvial y el llegar a determinar el curso causante de dichos depósitos y su relación con el actual Esva o Canero, así como explicar el cauce, en la actualidad seco, que forma la playa de Bozo, son alguno de los interrogantes que se tienen planteados en cuanto a la reconstrucción geomorfológica de la zona se refiere y que estamos seguros que en sus respuestas esconden las explicaciones sobre el más antiguo y primitivo poblamiento humano y de sus paleoculturas.

Si tenemos presente el lugar estratigráfico dentro del nivel V., de donde se ha extraído el "canto - núcleo", pensamos que este momento debió ser antiguo dentro de la época en la que se formó el citado depósito.

Del análisis de la pieza se puede determinar la inexistencia de un fuerte rodamiento, con lo que cabe pensar que ésta no habría sido arrastrada desde zonas demasiado alejadas al lugar en el que se ha encontrado, por lo que consideramos este resto como indicio de una "ocupación" en la Rasa litoral, que en esta zona tiene no más de tres $\mathrm{Km}$. de ancho. En algún lugar de ella, cercano al sitio de Busto, seguramente descansan los restos de un posible asentamiento humano, del cual el río citado arrastró algunos, uno de los cuales es el "canto-núcleo" por nosotros recuperado.

La formación de la Rasa en momentos no muy antiguos, más probablemente durante el pleistoceno inferior que durante el plioceno (piénsese que faltan completamente niveles sedimentológicos asignables al plioceno y al pleistoceno inferior), la interpretación fluvial de los depósitos existentes en la actual línea de costa y la existencia de materiales tallados antrópicamente intercalados en estos niveles son los enunciados de las más importantes conclusiones a las que hemos llegado tras las excavaciones del yacimiento de Busto. 


\section{BIBLIOGRAFÍA}

ÁLVAREZ, M. A. y DÍAZ - FIERROS, F.(1995): "Los suelos”. Geologia de Asturias. Ed. Trea. S.L. 173-186. ARAMBURU, C. y BASTIDA, F. (Editores) (1995): Geología de Asturias. Ed. Trea.

ASENSIO, I. (1970): "Rasgos geomorfológicos de la zona litoral galaico astúrica en relación con las oscilaciones glacio eustáticas”. Estudios Geológicos. vol. XXVI. no 1.: 88 - 89. Madrid.

BARROIS, CH. (1882): Recherches sur les terrains anciens des Asturies et de la Galice. These. Mem. S. G. du Nord. vol. 2. n⿳⺈ 1

BIROT, P. y SOLÉ SABARÍS, L. (1954): Recherches morphologiques dans le Nord-Ouest de la Peninsule Iberique. Publi. del Instituto Geológico. Univ. Barcelona.

CABRERA, V. (1984): El yacimiento de la cueva de El Castillo. Bibliotheca Prehistórica Hispánica. vol. XXII. Madrid.

CANO PAN, J.A. \& VÁZQUEZ VARELA, J.M. (1996): "An ethnografic model for the flaking of quartzite artefacts". in Moloney, N. Raposo.L. y Santonja M. (Eds) : Non-Flint Stone Tools and the Paleolithic Occupation of the Iberian Peninsula. B. A. R. International Series 649.

DÍAZ NOSTY, B. \& SIERRA, G. (1995): "Carta Arqueológica del concejo de Cudillero" Excavaciones Arqueológicas en Asturias 1991-1994: 190-191. Oviedo

_- (1995): "Carta Arqueológica del concejo de Soto del Barco" Excavaciones Arqueológicas en Asturias 1991-1994: 198-199. Oviedo

FLOR, G. (1983): “Las Rasas asturianas: Ensayos de correlación y emplazamiento”. Trabajos de Geología, 13: 65 - 81. Univ. de Oviedo,

GAMBLE, C. S.(1990) : El poblamiento paleolítico de Europa. Ed. Crítica. Barcelona. Trad. Castellana.

GÓMEZ DE LLARENA, J. (1933): "Datos de Historia Natural de Asturias" Reseñas Científicas de la Sociedad española de Historia Natural, VIII: 157. Madrid

— y ROYO GÓMEZ, I. (1927): "Las terrazas y rasas litorales de Asturias y Santander". Bol. de la Sociedad de Historia Natural., XVII: 19-38. Madrid.

GONZÁLEZFERNÁNDEZ, J.M. (1968): "El Paleolítico inferior y medio en Asturias". Archivum, XVIII: 1-36. Oviedo

GUILCHER, A. (1977): "Les "rasas": Un probleme de morphologie littorale generale". Annales de Geographie. no 455. Anne LXXXIII: 1 - 33.

GUITIAN, F., MUÑOZ, M., CARBALLAS, T. y ALBERTO, F. (1985): Suelos naturales de Asturias. C.S.I.C. Instituto de Investigaciones Agrobiológicas de Galicia. Santiago de Compostela

HERNÁNDEZ PACHECO, E. (1930): Mouvements et dépôts sur les côtes d'Espagne pendant le Pliocene et Pleistocene. 2eme. Raport. Col. Temas Plio-Pleistoceno. Florencia.

- (1932): Síntesis fisiográfica y geológica de España. Trab. Museo Nacional Ciencias Naturales. Serie Geológica no 38 Madrid.

(1933): "Le probleme des terrasses Pliocenes et Pleistocenes". Congreso de París.

(1939): "Les cavernes préhistoriques de la côte cantabrique comme preuve de la stabilité du littoral". Mélanges de Préhistoire et d'Anthropologie offerts au professeur Comte H. Begouen. Toulouse.

HERNÁNDEZ PACHECO, F. y ASENSIO AMOR, I. (1959): "Materiales sedimentarios sobre la Rasa cantábrica". Bol. R. S. Española de Historia Natural., 57: 75 - 100.

(1960): "Materiales sedimentarios sobre la Rasa cantábrica". Bol. R. Soc. Española de Historia Natural., 58: $73-83$.

- (1961): "Materiales sedimentarios sobre la Rasa Cantábrica". Bol. R. Soc. Española de Historia Natural., 59 pp. $207-223$. 
- (1962): Materiales sedimentarios sobre la Rasa cantábrica". Bol. R. Soc. Española de Historia Natural., 60: $65-76$.

- (1963): "Materiales sedimentarios sobre la Rasa cantábrica". Bol. R. Soc. Española de Historia Natural., 61: $89-120$.

- (1964): "Recientes investigaciones sobre la génesis de la rasa cantábrica". Bol. R. Soc. Española de Historia Natural., 62: 61 - 89.

HOYOS, M. (1995): "Secuencia estratigráfico-geológica del paleolítico inferior en la cornisa cantábrica". Jornadas sobre el primer poblamiento humano en la cornisa cantábrica. (comunicación oral). Luarca. JORDÁ, F. (1967): "La España de los tiempos paleolíticos". Las Raíces de España. Instituto Español de Antropología Aplicada.. 1 - 26. Madrid.

LLOPIS, N. (1953): "Los depósitos de la costa cantábrica entre los cabos Busto y Vidío (Asturias)". Speleon., VI: 333 - 347. Oviedo.

(1957): "La plataforma costera de la costa asturiana entre el cabo Busto y el Eo y sus depósitos". Com.

V. Congres. Inter. I.N.Q.U.A. Madrid - Barcelona.

(1963): "Estudio geológico de los alrededores de Luarca. (Asturias)". Bol. Instituto Geológico y Minero de España. t. LXXIV: 15 - 83. Madrid

- y JORDÁ, F.(1957): Mapa del Cuaternario de Asturias. V. Congreso Internacional I.N.Q.U.A. Oviedo. MARADONA, J. A. y MARTÍNEZ FAEDO, L. (1995): “ Inventario arqueológico del concejo de Tapia de Casariego" Excavaciones Arqueológicas en Asturias. 1991-1994: 174 - 175. Oviedo

MARCOS, A. (1976): Mapa geológico de España. E: 1:50.000. Busto. I.G.M.E. Madrid

MARY, G. (1971): "Les hautes surfaces d'abrasión marine de la côte asturienne (Espagne)". Histoire structurale du Golfe de Gascogne. t. 2 vol. 5: 1 - 12. París.

MONTES, R. y FERNÁNDEZ RAMOS, M. (1997. e.p.): "El bifaz de Cancienes (Corvera de Asturias): Un nuevo hallazgo lítico de tipología inferopaleolítica en el centro de la región asturiana".Real Instituto de Estudios asturianos. Boletín de Ciencias. Oviedo.

MUÑOZ JIMÉNEZ, J. (1985) : "Geografía Física". Geografía de Asturias. vol. 1. Ed. Ayalga. Oviedo. NONN, H. (1966): Les régions côtières de la Galice (Espagne). Etude géomorphologique. C.N.R.S. París.

NOVAL, M. (1995): "Seguimiento arqueológico de la autovía Oviedo-Pola de Siero. 1990-1991" Excavaciones Arqueológicas en Asturias. 1991-1994 : 253-257 Oviedo

(1996): Informe seguimiento arqueológico tramo Novellana-Cadavedo. C-634. Oviedo

PÉREZ PÉREZ, M. (1975): "Los yacimientos prehistóricos de la región del cabo Peñas" XIII Congreso Nacional de Arqueología : 109-118. Zaragoza

— y GONZÁLEZ MENÉNDEZ, L. (1990): "El yacimiento paleolítico de Santa María del Mar (Castrillón. Asturias) y características geológicas del entorno" Boletín del Real Instituto de Estudios Asturianos, 135: 591-615. Oviedo

(1991): "El yacimiento paleomesolítico de "Pinos Altos"'-Santa Martín de Laspra, Castrillón (Asturias) y su entorno geológico ambiental" Real Instituto de Estudios Asturianos. Boletín de Ciencias, 41:275-344. Oviedo

(1996): "Nuevo yacimiento paleolmesolítico en Aramar, Luanco (Gozón. Asturias) y sus aspectos geológicos" Veleia, 13: 7-70. Vitoria

RODRÍGUEZ ASENSIO, J.A.(1976): "Bifaz achelense de Busto. (Luarca)". Boletín del Instituto de Estudios Asturianos, 87: 217 - 230. Oviedo

(1978): "Nota preliminar sobre las excavaciones del yacimiento de Bañugues (Gozón. Asturias)". Boletín del Instituto de Estudios Asturianos, 93-94: 357 - 368. Oviedo

_ (1978): "The Early Paleolithic site of Bañugues (Gozón. Asturias. Spain)”. Current Anthropology, 19. $\mathrm{n}^{\mathrm{0}} 3: 615-616$. 
(1980):'Bañugues (Gozón. Asturias). Yacimiento del paleolítico inferior". Noticiario Arqueológico Hispánico., 9: 11 - 31. Madrid.

- (1983): La presencia humana mas antigua en Asturias. Estudios de Arqueología Asturiana noํ2. Oviedo. (1983): "Excavaciones en el yacimiento de Paredes (Siero. Asturias) y los yacimientos del paleolítico inferior en la cuenca media de los ríos Nora y Noreña" Noticiario Arqueológico Hispánico, 15: 9-37. Madrid

(1995): "Excavaciones Arqueológicas en Cabo Busto (Valdés). Un asentamiento achelense". Excavaciones Arqueológicas en Asturias. 1990 - 94. : 7-18. Oviedo.

(1996): "Analysis of Quartzites as implement blanks in the Early Paleolithic of Asturias, Northern Spain”. in Moloney, N. Raposo.L. y Santonja M. (Eds) : Non-Flint Stone Tools and the Paleolithic Occupation of the Iberian Peninsula. B. A. R. International Series 649. pp. 33 - 36.

(1996): "Estratigrafía del yacimiento de cabo Busto (Valdés). Una secuencia del Pleistoceno medio en Asturias". Boletín del Real Instiruto de Estudios Asturianos, 148: 367 -383. Oviedo

(1997 e.p.): "Yacimiento de Louselas (Ribadeo. Lugo). Un conjunto lítico del paleolítico antiguo". Fervedes, 4. Villalba. Lugo

RODRÍGUEZ ASENSIO, J. A. y FLOR, G.(1980): "Estudio del yacimiento prehistórico de Bañugues y su medio de depósito (Gozón. Asturias)". Zephyrus, XXX - XXXI: 205 - 222. Salamanca.

- (1983): "Industrias paleolíticas eolizadas de la región del cabo Peñas". Cuadernos de laboratorio xeoloxico de Laxe, 5: 23 - 46. A Coruña

RODRÍGUEZ ASENSIO, J. A. y NOVAL, M. A. (1997 e.p.): El Paleolítico Antiguo en Gijón. Breve aproximación a los primeros grupos predadores en la Prehistoria de Asturias. Gijón

STRINGER, C. y GAMBLE, C.(1996): En busca de los Neandertales. La solución al rompecabezas de los orígenes humanos. Ed. Crítica / Arqueología. Barcelona.

SCHULZ, G. (1858): Descripción geológica de la provincia de Oviedo. Madrid. 


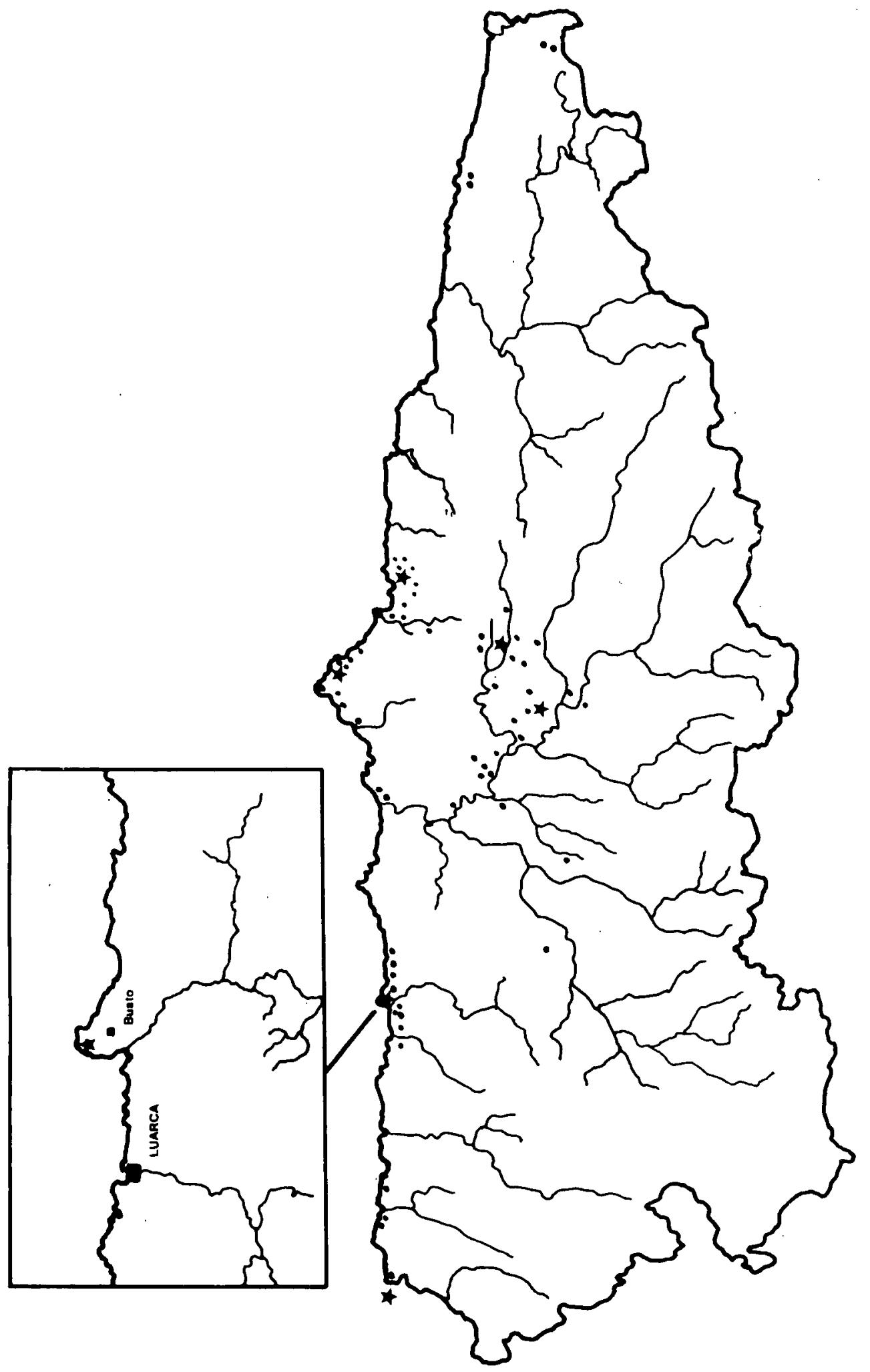

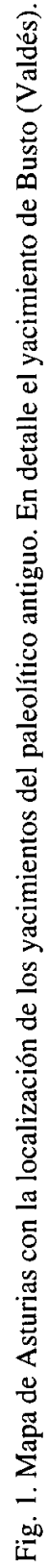


0 mts.

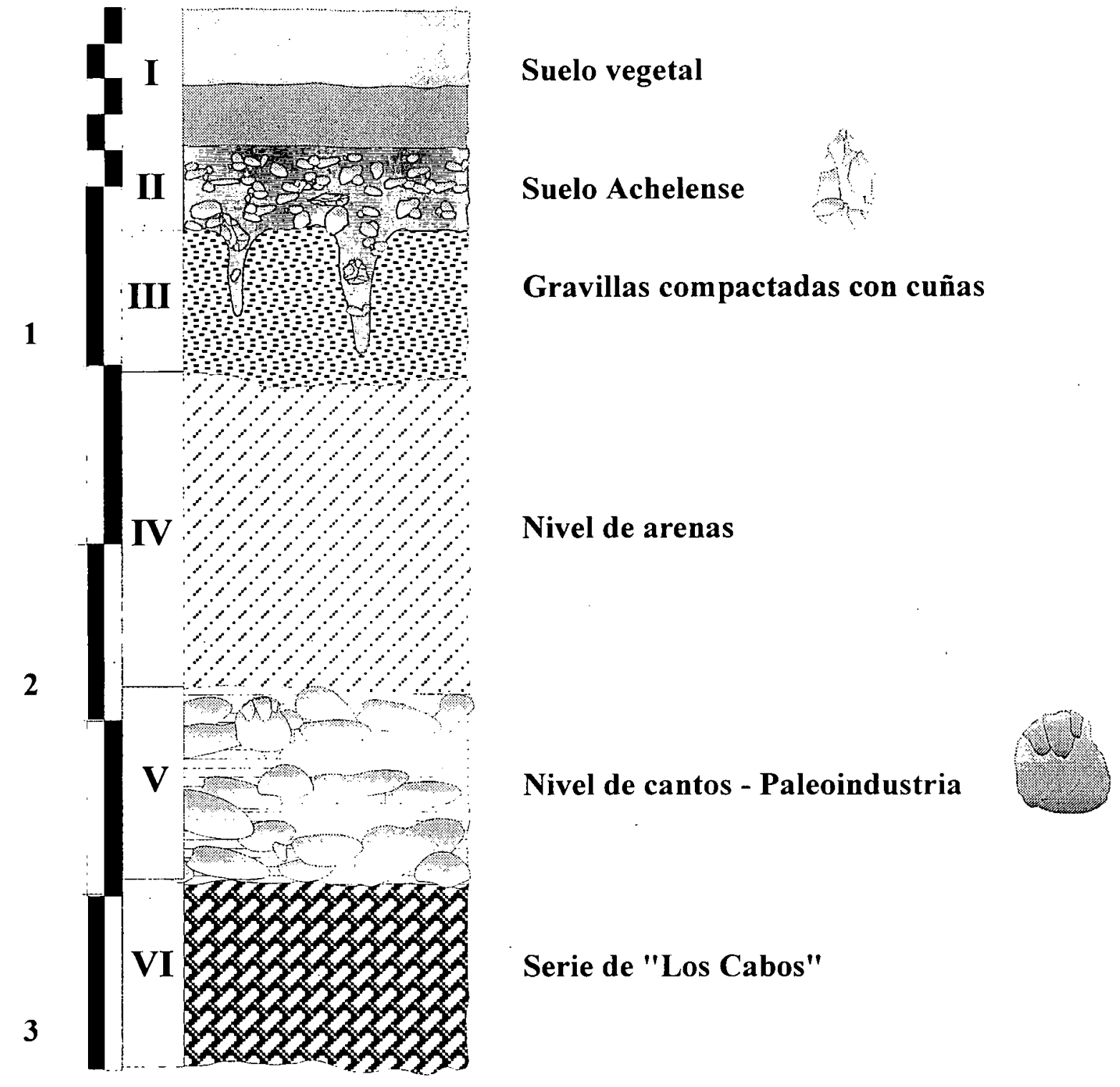

\section{Yacimiento de Cabo Busto (Valdés) Corte estratigráfico}

Fig. 2. Reconstrucción gráfica de la estratigrafía del yacimiento de Busto. En ella se pueden apreciar los dos niveles con industrias prehistóricas [achelense superior (II) y paleoindustrias (V)]. 

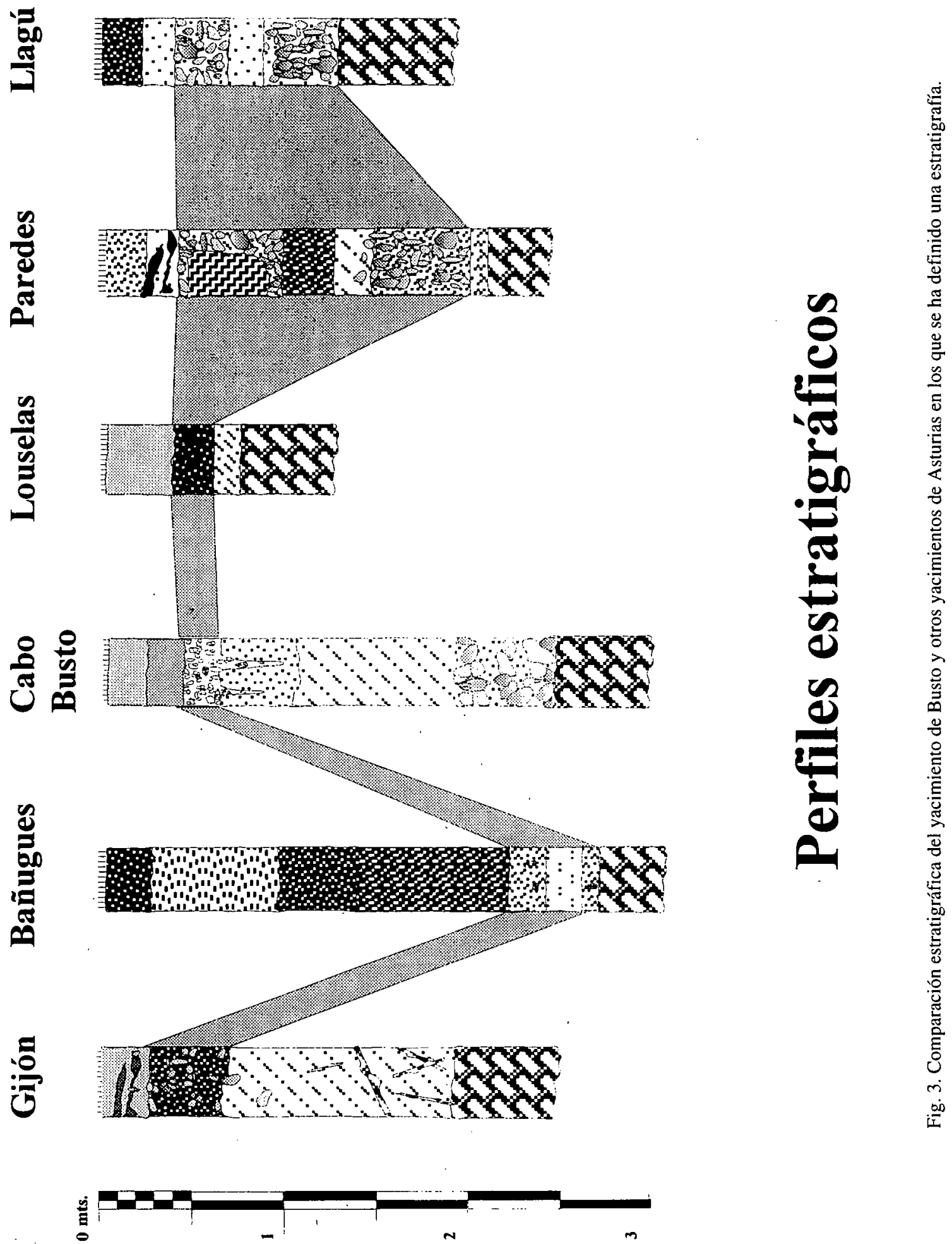

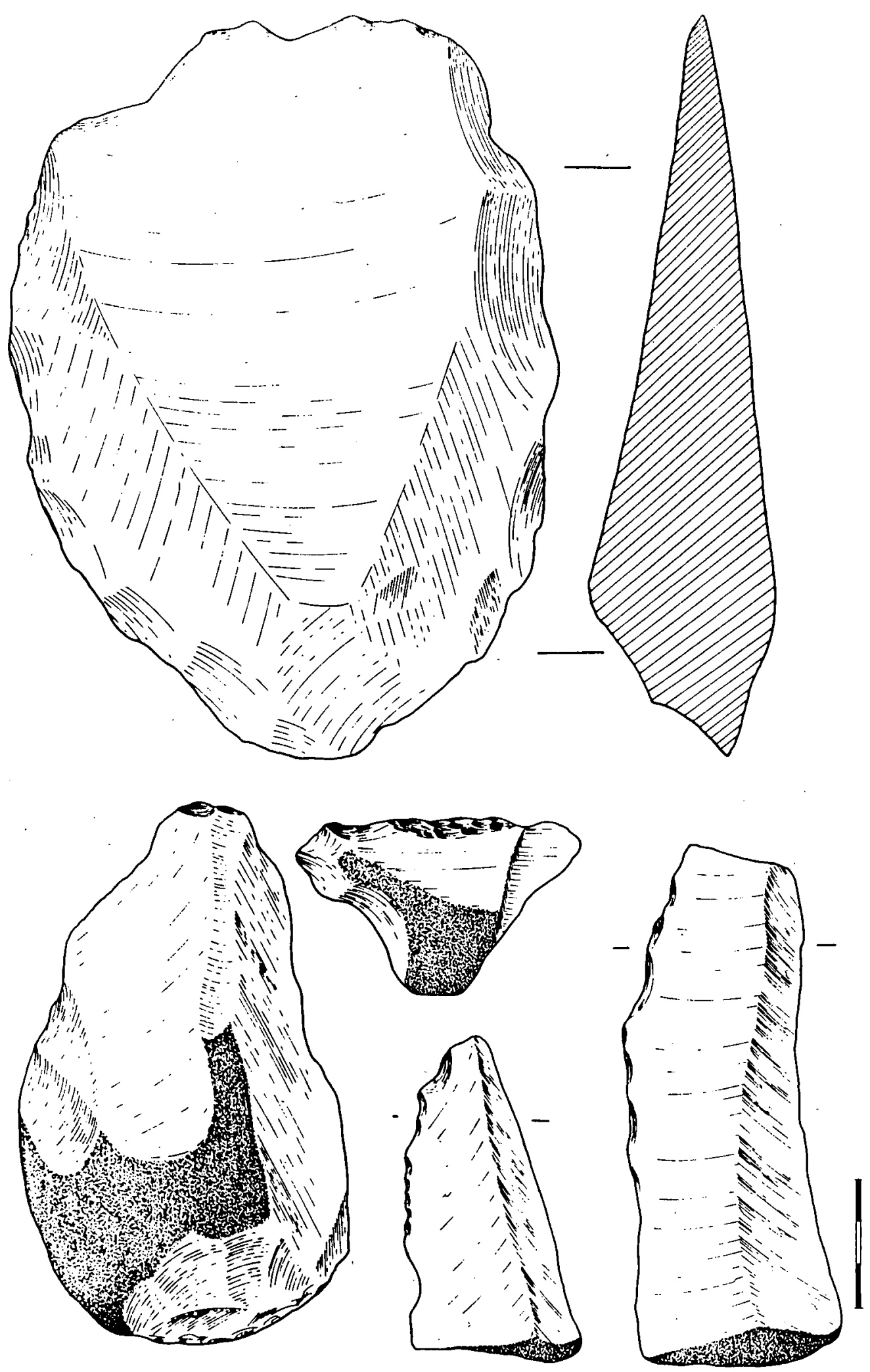

Fig. 4. Materiales líticos del nivel II 


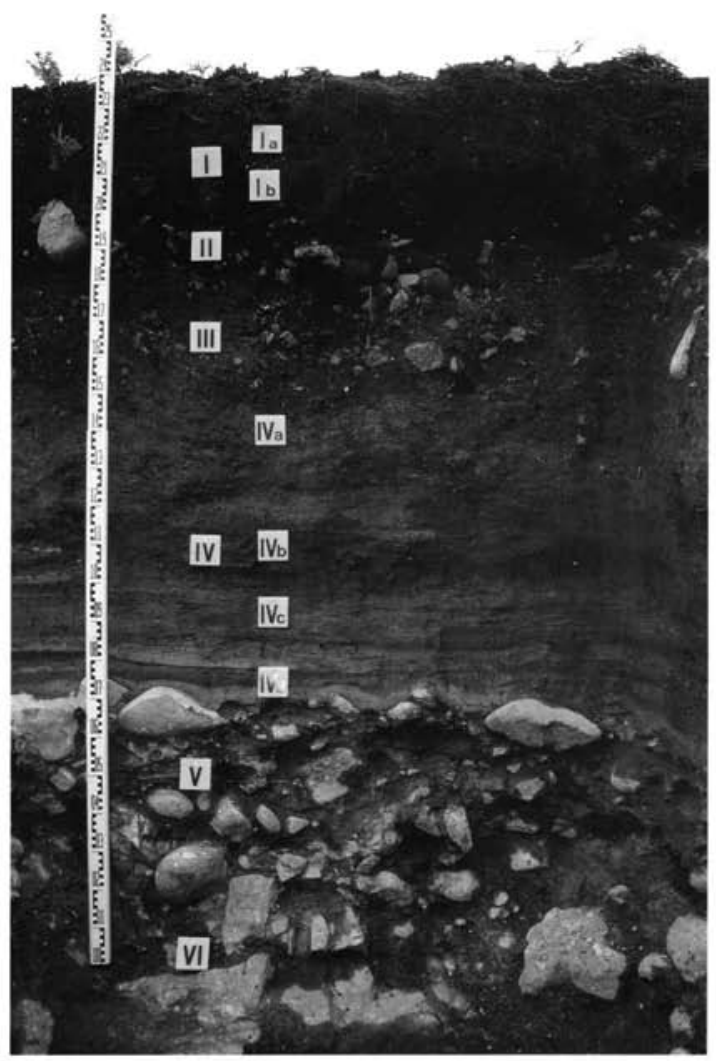

Lám. I. Estratigrafía del yacimiento de cabo Busto tras las excavaciones arqueológicas llevadas a cabo 


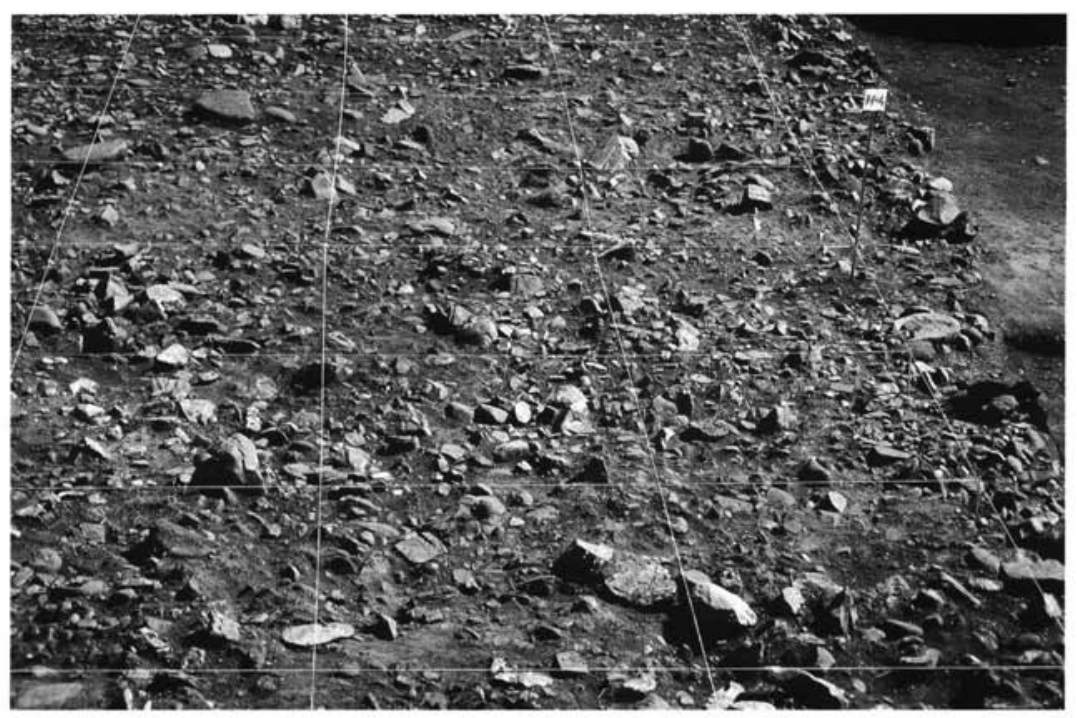

Lám. II. Momento de excavación del nivel II. 


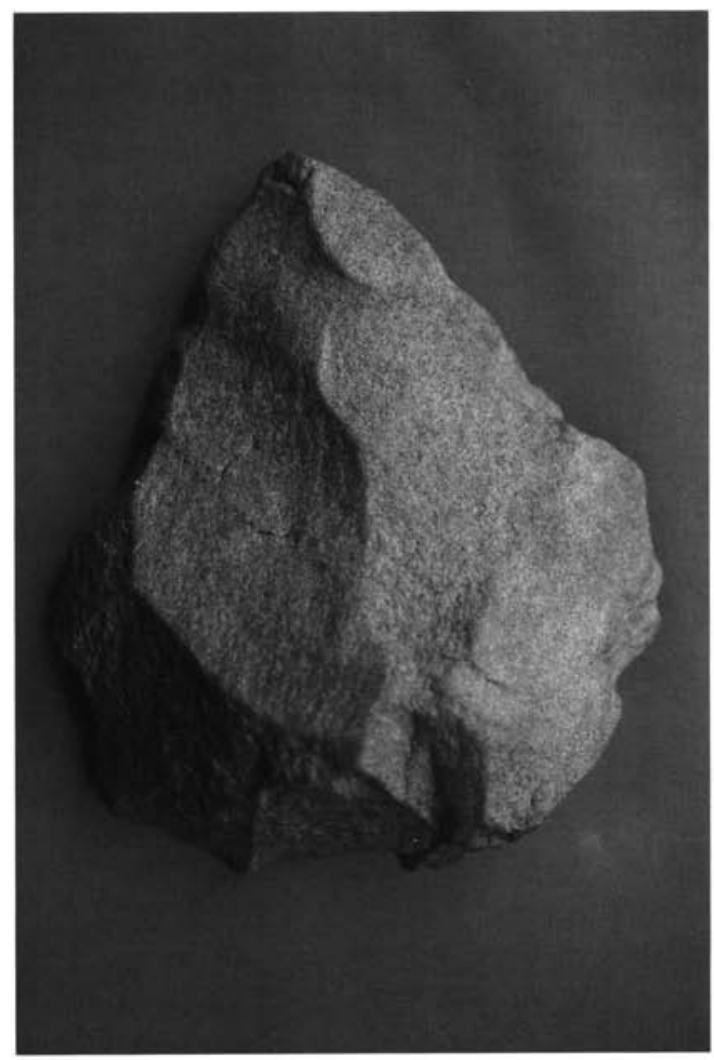

Lám. III. Bifaz del nivel achelense superior 


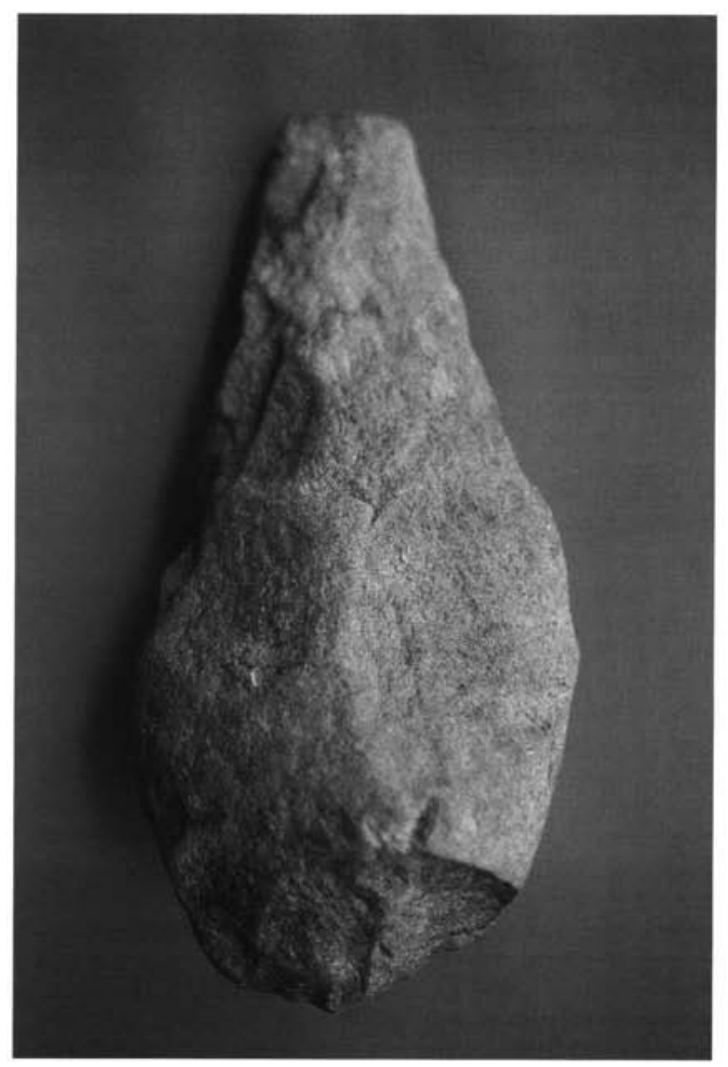

Lám. IV. Bifaz del nivel achelense superior 


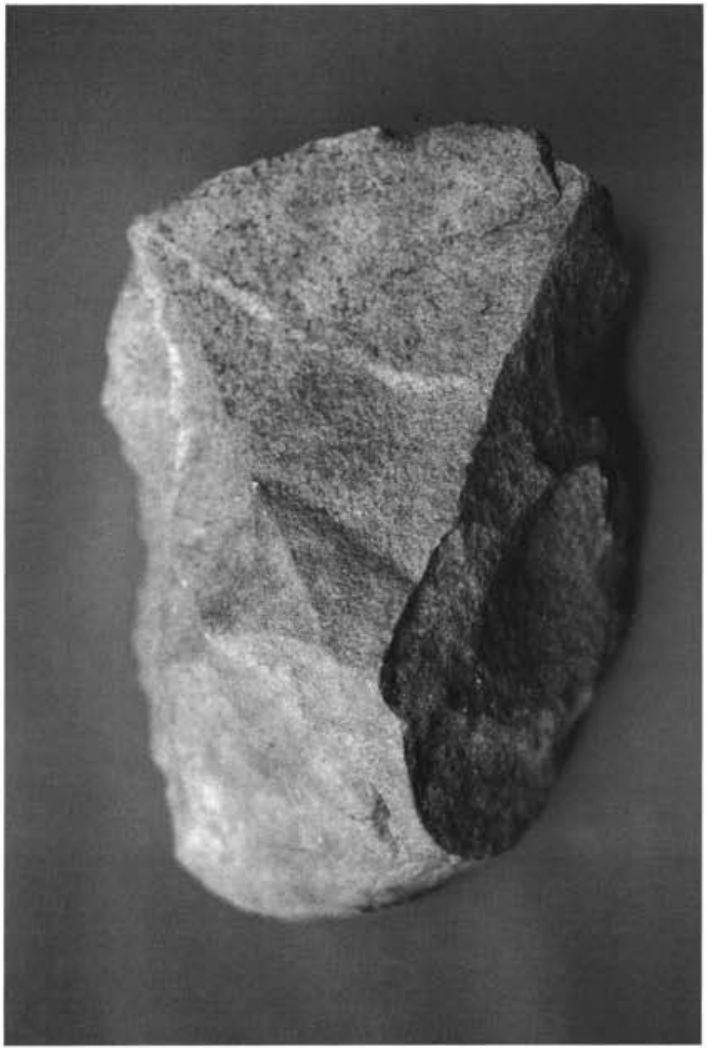

Lám. V. Hendidor del nivel achelense superior 


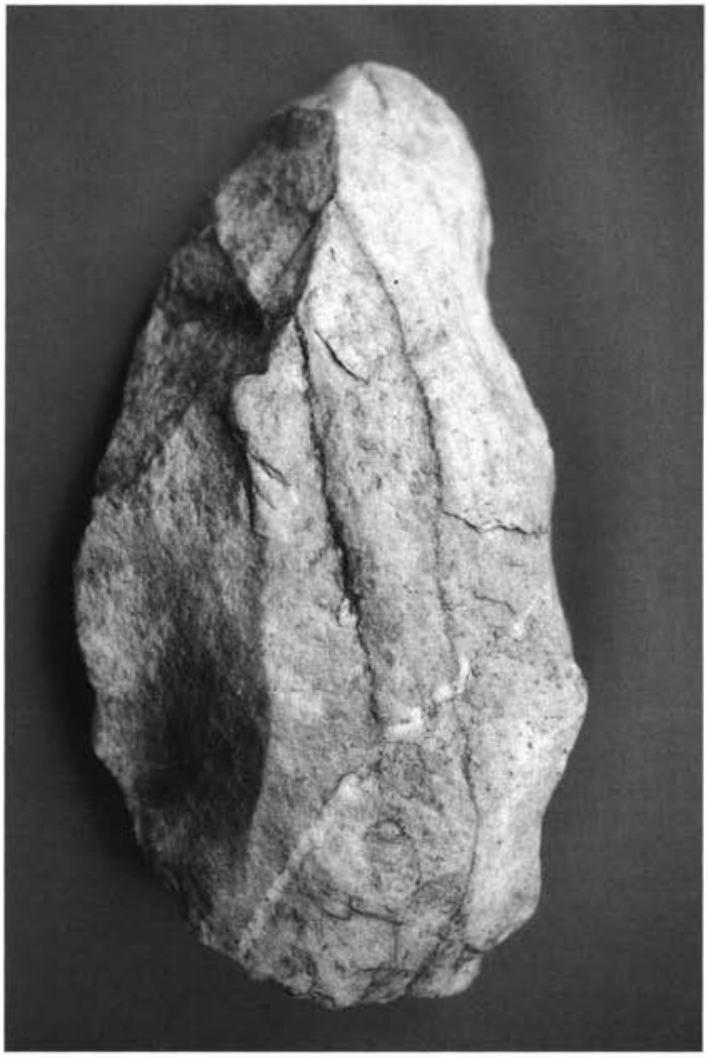

Lám. VI. Pico triedro del nivel achelense superior 


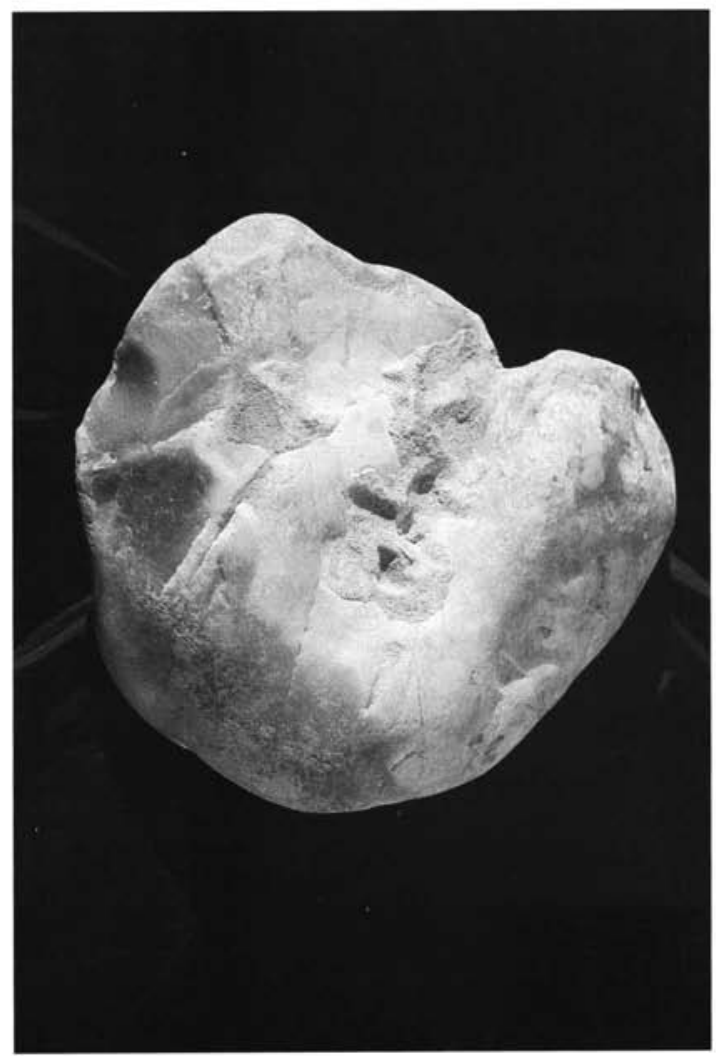

Lám. VII. Canto trabajado (¿núcleo?) del nivel V (paleoindustrias) 


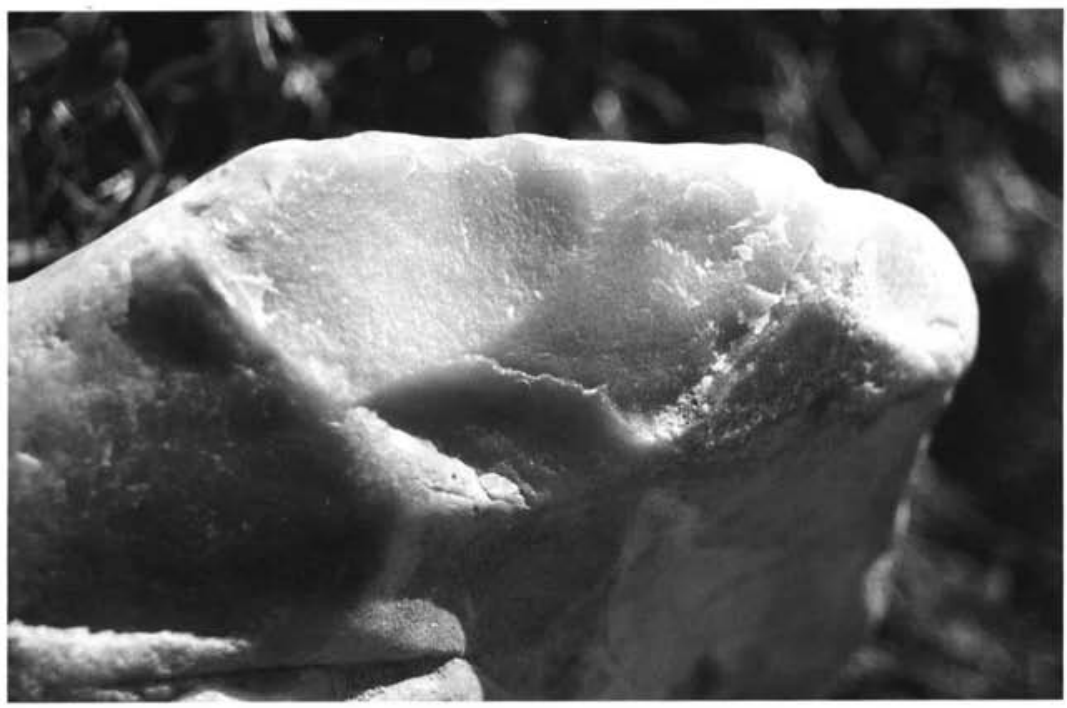

Lám. VIII. Detalle de los levantamientos del canto trabajado del nivel V 


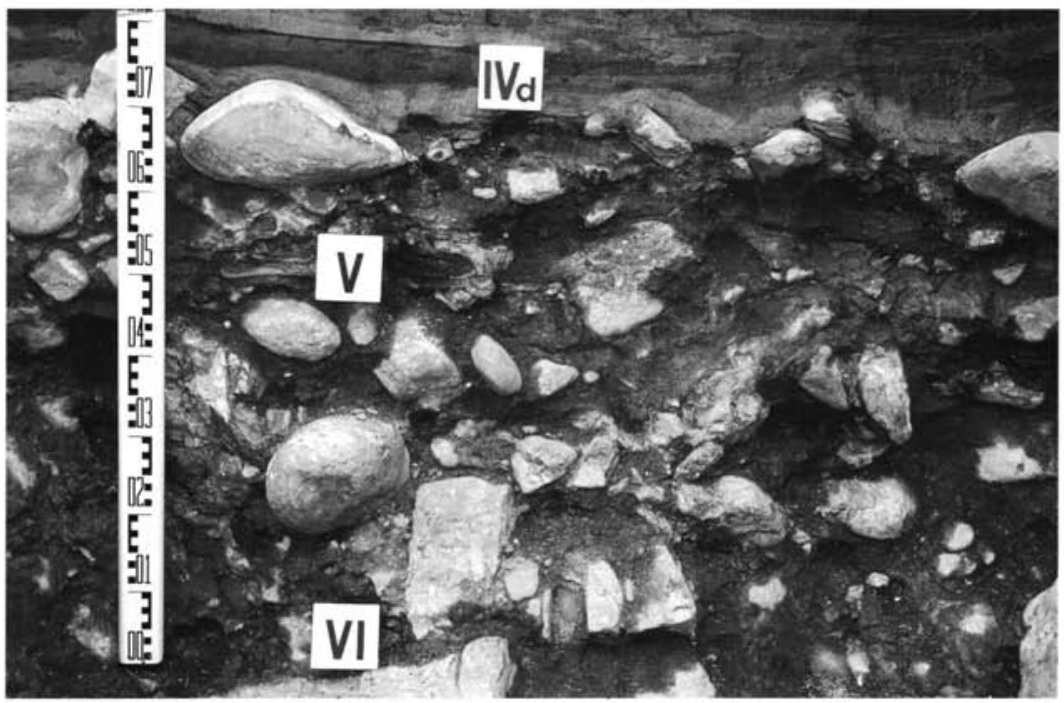

